\title{
Blood Flow in Prefractal Media: Rheological Model Approaches and Sensitivity Analysis
}

\author{
A. F. Miguel \\ Department of Physics, School of Science and Technology, University of Evora, Evora, Portugal \\ Institute of Earth Sciences (ICT), Pole of Evora, 7000-671, Evora, Portugal \\ †Corresponding Author Email: afm@ uevora.pt
}

(Received March 29, 2020; accepted May 18, 2020)

\begin{abstract}
The development of microfluidic media supporting blood flow is significant for many applications. Prefractal models have considerable potential for contributing to the study of flow in these media, since information about scale complexity is captured by a small number of parameters. Flows of power law fluids, Bingham fluids and described by the marginal zone theory are considered. In this study, physically based models for estimating the permeability of a microfluidic porous materials are presented. Models are derived assuming that media are represented by a bundle of tortuous capillary tubes with fractal pore-size distributions. They are expressed in terms of porosity, microstructural parameters and fluid characteristics. Expressions for the flow resistance through single tortuous tubes, and the relationship between fluid velocity through tortuous tubes and through straight tubes, in terms of fractal dimensions, are also obtained.
\end{abstract}

Keywords: Tortuous capillaries; Flow resistance; Permeability; Blood flow; Fractal; Prefractal.

\section{NOMENCLATURE}

$D_{d} \quad$ fractal dimension for tubes diameter

$D_{L} \quad$ fractal dimension for tubes length

$K \quad$ permeability of the porous material

$k_{B p} \quad$ consistency index Bingham plastic fluid

kow $\quad$ consistency index power law fluid

$L_{0} \quad$ straight-line length

$p \quad$ pressure

$R \quad$ flow resistance

$u \quad$ fluid velocity

$\mu_{c} \quad$ fluid viscosity at central region of the tube $\mu_{z} \quad$ plasma viscosity

$\tau \quad$ shear stress

$\tau_{0} \quad$ yield stress

$\phi \quad$ volumetric flow rate

$\chi \quad$ tube diameter

$\chi_{c} \quad$ diameter of the marginal zone

$\chi_{\max } \quad$ maximum diameter

$\chi_{\min } \quad$ minimum diameter

$\chi_{p} \quad$ diameter of the plug

$\omega \quad$ fluid behavior index

\section{INTRODUCTION}

Blood flow supplies the body's cells with enough oxygen and nutrients required to operate. This essential function is accomplished by vessels with different sizes, some of them are straight and others are tortuous or twisted (Dougherty and Varro 2000; Fortier et al. 2014), forming a complex spatial organization. Blood vessels vary in size. For blood flow in larger vessels, where the typical shear rate exceeds $100 \mathrm{~s}^{-1}$, a Newtonian model constitute a good approximation for blood. In smaller vessels, the non-Newtonian behavior manifests and must be accounted (Fung 1993). The Fåhraeus-Lindqvist effect (Fåhræus and Lindqvist 1931) is associated with the concentration of red blood cells in the core region of small vessels surrounded by a cell-free marginal layer (i.e., the annular region is a cell-free layer). Continuum fluid assumption may break down as the diameters of the vessels is of order of magnitude of red blood cells (Pries et al. 1992; Haynes 1960).

Bodily blood flows are complex but seem to show a statistical scale-invariance. Therefore, some studies point to the fact that the complex organization of vessels can be studied based on the concept of 
fractal (Bittner 1991; Sandau and Kurz 1997; Masters 2004). In fact, they are only fractal over a finite range of scales and should be called as prefractals.

Blood vessel arrangement patterns can be likened to porous media (Bejan et al. 2004). Pore network controls the hydrodynamics characteristics of these media. Applying fractals to describe these configurations is very attractive because complexity, at different scales, is defined by a small number of parameters. Therefore, fractal geometry has a great potential to provide a convenient description of these media (e.g., Katz and Thompson 1985; Jacquin and Adler 1987; Krohn 1988; Adler 1996; Wei et al. 2015; Lei et al. 2019), including the analysis of flow properties. Permeability is a key property which expresses the ability of fluid to flow through the medium, and may be related to the fractal dimension (e.g., Hansen and Skjeltorp 1988; Miguel et al. 2000; Yu and Cheng 2002; Yun et al. 2010; Miguel 2010; Tana et al. 2014; Cai et al. 2015; Tan et al. 2017).

Microfluidic blood flow devices are composed of networks of tubes with sizes similar to blood vessels. These devices emulating body flows and have a potential to yield biomedical technologies (Park et al. 2005; Illa et al. 2014; Chen et al. 2016; Delalat et al. 2018). The development of these devices requires similar design to emulating fluid flow.

This paper study the fundamental problem of blood flow through fractal media composed by bundles of tortuous tubes. As fluidic environments with different sizes restrict the fluid to a particular rheological regime, different rheological models for blood are considered and compared.

\section{LaMinar BloOd Flow Through Capillary Tubes}

Many suspensions of solid particles or macromolecules are described by a power-law model. The Ostwald de Waele model expresses that

$\tau=\mathrm{k}_{\mathrm{ow}}\left(\frac{\mathrm{du}}{\mathrm{d} \chi}\right)^{\omega}=-\frac{\chi}{4} \frac{\Delta \mathrm{p}}{\mathrm{L}}$

where $\tau$ is the shear stress, $u$ is the fluid velocity, $p$ is the pressure, $\mathrm{k}_{\text {ow }}$ is the consistency index, and $\omega$ is the fluid behaviour index. Integrating this equation with respect to $\chi$, and defining the volumetric flow rate $\phi$ as the product of the axial velocity and the cross-sectional area of the tube, yields (Miguel 2016)

$\phi(\chi)=\frac{\pi \omega}{8(3 \omega+1)} \chi^{\frac{3 \omega+1}{\omega}}\left(\frac{1}{4 \mathrm{k}_{\mathrm{ow}}} \frac{\Delta \mathrm{p}}{\mathrm{L}}\right)^{\frac{1}{\omega}}$

It should be pointed out that for $\omega<1$ the fluid exhibits shear-thinning properties, whereas for $\omega>1$ the fluid exhibits shear-thickening behavior. The constitutive equation of Newtonian fluid is recovered for $\omega=1$. For blood $\omega$ lies between 0.56 and 0.75 (Fung 1993).

Bingham plastic model accounts for a plug region in which the shear stress is less than the yield stress and the constitutive equation is

$\tau=\tau_{\mathrm{o}}-\mathrm{k}_{\mathrm{Bp}} \frac{\mathrm{du}}{\mathrm{d} \chi}$

where $\tau_{0}$ is the yield stress, and $\mathrm{k}_{\mathrm{Bp}}$ is the consistency index for the Bingham plastic fluid. Consider that the diameter of the plug $\left(\chi_{p}\right)$ is much lower than the diameter of the tube $(\chi)$. According to the Buckingham-Reiner equation (Bird et al. 1960), the volumetric flow rate is given by

$\phi(\chi)=\frac{\pi}{128 \mathrm{k}_{\mathrm{Bp}}} \chi^{4} \frac{\Delta \mathrm{p}}{\mathrm{L}}\left(1-\frac{4}{3} \frac{\chi_{\mathrm{p}}}{\chi}\right)$

with

$\chi_{\mathrm{p}}=\frac{4 \tau_{\mathrm{o}} \mathrm{L}}{\Delta \mathrm{p}}$

Note that for a null yield stress, Eq. (4) reduces to the Hagen-Poiseuille equation.

To account for the Fahraeus-Lindquist effect, the marginal zone theory (Haynes 1960) suggests that the blood flow through a tube is obtained by applying Hagen-Poiseuille law to the central and to the marginal zone of flow, and so the total volumetric flow rate is given by

$\phi(\chi)=\frac{\pi}{128 \mu_{\mathrm{z}}} \chi^{4} \frac{\Delta \mathrm{p}}{\mathrm{L}}\left[1-\vartheta\left(1-\frac{\chi_{\mathrm{c}}}{\chi}\right)^{4}\right]$

with

$\vartheta=\left(1-\frac{\mu_{\mathrm{z}}}{\mu_{\mathrm{c}}}\right)$

Here $\mu_{\mathrm{z}}$ is the plasma viscosity, $\mu_{\mathrm{c}}$ is the viscosity of the fluid in the central region of the tube, and $\chi_{\mathrm{c}}$ is diameter of the marginal zone. Equation (5) is used to blood flows through vessels with diameters ranging from 4 to $500 \mu \mathrm{m}$. Note that the HagenPoiseuille equation is recovered when $\chi=\chi_{c}$ or $\mu_{\mathrm{p}}=\mu_{\mathrm{c}}$.

\section{FRACTAL MODEL FOR BLOOD FLOW THROUGH CAPILLARY TUBES}

\subsection{Fractal Bundle of Tortuous Capillary Tubes}

Consider a porous material composed by a bundle of $\mathrm{N}$ tortuous capillary tubes with variable crosssectional areas. Let $\chi$ be the diameter of the capillary tubes, and $\mathrm{L}$ the length of the capillary tubes. Assuming that the length of tubes exhibits a fractal behaviour with respect to $\chi$ (Pitchumani and Ramakrishnan 1999; Miguel et al. 2000) 


$$
\mathrm{L}(\chi)=\frac{\mathrm{L}_{0}^{\mathrm{D}_{\mathrm{L}}}}{\chi^{\mathrm{D}_{\mathrm{L}}-1}}
$$

where $\mathrm{L}_{0}$ is the straight-line length, and $\mathrm{DL}_{\mathrm{L}}$ is the fractal dimension for tubes lengths.

The cumulative tube distribution follows the power law (Pitchumani and Ramakrishnan 1999; Miguel et al. 2000)

$$
\mathrm{N}(\mathrm{L} \geq \chi)=\left(\frac{\chi_{\max }}{\chi}\right)^{\mathrm{D}_{\mathrm{d}}}
$$

where $\chi_{\max }$ and $D_{d}$ are the maximum diameter and the fractal dimension for tubes diameters, respectively.

According to Eqs. (6) and (7), the variation of length of tubes and number of tubes, lying within the infinitesimal interval $\chi$ to $\chi+\mathrm{d} \chi$, is given by (Pitchumani and Ramakrishnan, 1999; Miguel et al. 2000)

$$
\begin{aligned}
& \mathrm{dL}=\mathrm{D}_{\mathrm{L}}\left(\frac{\mathrm{L}_{0}}{\chi}\right)^{\mathrm{D}_{\mathrm{L}}-1} \mathrm{dL}_{0} \\
& -\mathrm{dN}(\mathrm{L} \geq \chi)=\mathrm{D}_{\mathrm{d}} \frac{\chi_{\max }^{\mathrm{D}_{\mathrm{d}}}}{\chi^{\mathrm{D}_{\mathrm{d}}+1}} \mathrm{~d} \chi
\end{aligned}
$$

Note that the length of the tubes decreases with $\chi$, and since the fractal dimension $\mathrm{D}_{\mathrm{L}}=1$ the porous material is made of straight capillary tubes (Eq. 6). In addition, the increase of size of the tubes decreases the number of tubes (Eq. 9).

According to Eq. (7), the total number of tubes from $\chi_{\min }$ to $\chi_{\max }$ is given by

$$
\mathrm{N}\left(\mathrm{L} \geq \chi_{\min }\right)=\left(\frac{\chi_{\max }}{\chi_{\min }}\right)^{\mathrm{D}_{\mathrm{d}}}
$$

where $\chi_{\max }$ and $\chi_{\min }$ are the maximum and the minimum diameters, respectively.

Taking into account Eqs. (9) and (10) yields

$$
-\frac{\mathrm{dN}\left(\mathrm{L} \geq \chi_{\min }\right)}{\mathrm{N}\left(\mathrm{L} \geq \chi_{\min }\right)}=\mathrm{D}_{\mathrm{d}} \frac{\chi_{\min }^{\mathrm{D}_{\mathrm{d}}}}{\chi^{\mathrm{D}_{\mathrm{d}}+1}} \mathrm{~d} \chi
$$

Here $D_{d} \frac{\chi_{\min }^{D_{d}}}{\chi^{D_{d}+1}}$ is the probability of the pore diameter distribution. Integrating this distribution, the following condition must be satisfied

$$
\int_{\chi_{\min }}^{\chi_{\max }} \mathrm{D}_{\mathrm{d}} \frac{\chi_{\text {min }}^{\mathrm{D}_{\mathrm{d}}}}{\chi^{\mathrm{D}_{\mathrm{d}}+1}} \mathrm{~d} \chi=1-\left(\frac{\chi_{\min }}{\chi_{\max }}\right)^{\mathrm{D}_{\mathrm{d}}} \sim 1
$$

This condition holds for $\chi_{\min } / \chi_{\max } \leq 10^{-2}$ (Yu and Cheng 2002). This means that this formulation should be applied for this condition.

Besides, Eq. (6) allows us to relate the fluid velocity at the tortuous tube to fluid velocity at the straight tube, through the fractal dimension. Using the chain rule and Eq. (6), we obtain

$$
\mathrm{v}(\chi)=\frac{\mathrm{dL}(\chi)}{\mathrm{dt}}=\frac{\mathrm{dL}(\chi)}{\mathrm{dL}_{0}} \frac{\mathrm{dL}_{0}}{\mathrm{dt}}=\mathrm{D}_{\mathrm{L}}\left(\frac{\mathrm{L}_{0}}{\chi}\right)^{\mathrm{D}_{\mathrm{L}}-1} \mathrm{v}_{0}
$$

where $\mathrm{v}(\chi)$ is the fluid velocity at the tortuous tube and $\mathrm{v}_{0}$ is the fluid velocity at the straight tube. Note that since $\mathrm{L}_{0}>\chi, \mathrm{v}(\chi) / \mathrm{v}_{0}$ increases with increasing fractal dimension $\mathrm{D}_{\mathrm{L}}$.

\subsection{Fractal Blood Flow Through Capillary Tubes}

Let a bundle of tubes be composed by a number of not straight of tubes. For fractal tortuous tubes, introducing Eq. (6), to express the relationship between fractal dimension, $\mathrm{L}$ and $\mathrm{L}_{0}$, into Eqs. (2), (4) and (5), the fluid flow through a single tube is given by

$$
\begin{aligned}
& \phi(\chi)_{\mathrm{k}_{\mathrm{ow}}}=\frac{\pi \omega}{8(3 \omega+1)} \chi^{\frac{3 \omega+\mathrm{D}_{\mathrm{L}}}{\omega}}\left(\frac{1}{4 \mathrm{k}_{\mathrm{ow}}} \frac{\Delta \mathrm{p}}{\mathrm{L}_{0}^{\mathrm{D}_{\mathrm{L}}}}\right)^{\frac{1}{\omega}} \\
& \phi(\chi)_{\mathrm{k}_{\mathrm{Bp}}}=\frac{\pi}{128 \mathrm{k}_{\mathrm{Bp}}} \chi^{3+\mathrm{D}_{\mathrm{L}}} \frac{\Delta \mathrm{p}}{\mathrm{L}_{0}^{\mathrm{D}_{\mathrm{L}}}}\left(1-\frac{4}{3} \frac{\chi_{\mathrm{p}}}{\chi}\right) \\
& \phi(\chi)_{\mu_{\mathrm{z}}}=\frac{\pi}{128 \mu_{\mathrm{z}}} \chi^{3+\mathrm{D}_{\mathrm{L}}} \frac{\Delta \mathrm{p}}{\mathrm{L}_{0}^{\mathrm{D}_{\mathrm{L}}}}\left[1-\vartheta\left(1-\frac{\chi_{\mathrm{c}}}{\chi}\right)^{4}\right]
\end{aligned}
$$

Here Eqs. (14) to (16) represent the volumetric flow rate through a tortuous tube for power-law, Bingham plastic and marginal zone fluid models, respectively.

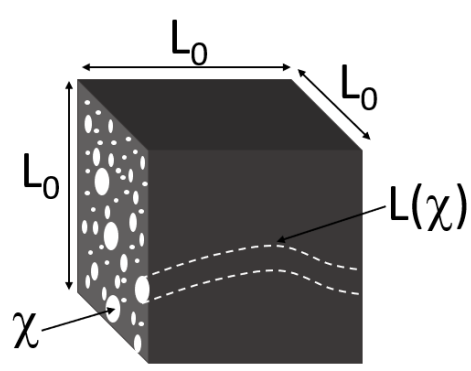

Fig. 1. Fractal porous material composed by capillary tubes. There are non-intersecting tubes which means no exchange of fluid between tubes.

We now consider a fractal porous media composed by tortuous and non-intersecting capillary tubes (Fig. 1). The total fluid flow $\Phi$ over the entire range of pore sizes (Eq. 7) can be expressed as

$$
\Phi=-\int_{\chi \min }^{\chi_{\max }} \phi(\chi)_{\tau} \mathrm{dN}(\mathrm{L} \geq \chi)=\int_{\chi \min }^{\chi_{\max }} \mathrm{D}_{\mathrm{d}} \frac{\chi_{\max }^{\mathrm{D}_{\mathrm{d}}}}{\chi^{\mathrm{D}_{\mathrm{d}}+1}} \phi(\chi)_{\tau} \mathrm{d} \chi
$$

By inserting Eqs. (14) to (16) into Eq. (17) yields

$$
\begin{aligned}
& \Phi_{\mathrm{k}_{\mathrm{ow}}}=\frac{\pi \omega \mathrm{D}_{\mathrm{d}} \chi_{\max }^{\mathrm{D}_{\mathrm{d}}}}{8(3 \omega+1)}\left(\frac{1}{4 \mathrm{k}_{\mathrm{ow}}} \frac{\Delta \mathrm{p}}{\mathrm{L}_{0}^{\mathrm{D}_{\mathrm{L}}}}\right)^{\frac{1}{\omega}} \int_{\chi_{\min }}^{\chi_{\max }} \chi^{\frac{2 \omega+\mathrm{D}_{\mathrm{L}}-\mathrm{D}_{\mathrm{d}} \omega}{\omega}} \mathrm{d} \chi \\
& \Phi_{\mathrm{k}_{\mathrm{pp}}}=\frac{\pi \mathrm{D}_{\mathrm{d}} \chi_{\max }^{\mathrm{D}_{\mathrm{d}}}}{128 \mathrm{k}_{\mathrm{Bp}_{\mathrm{p}}}} \frac{\Delta \mathrm{p}}{\mathrm{L}_{0}^{\mathrm{D}_{\mathrm{L}}}} \int_{\chi_{\min }}^{\chi_{\max }}\left(\chi^{2+\mathrm{D}_{\mathrm{L}}-\mathrm{D}_{\mathrm{d}}}-\frac{4}{3} \chi_{\mathrm{p}} \chi^{1+\mathrm{D}_{\mathrm{L}}-\mathrm{D}_{\mathrm{d}}}\right) \mathrm{d} \chi
\end{aligned}
$$


A. F. Miguel / JAFM, Vol. 13, No. 6, pp. 1675-1682, 2020.

$\Phi_{\mu_{z}}=\frac{\pi \mathrm{D}_{\mathrm{d}} \chi_{\max }^{\mathrm{D}_{\mathrm{d}}}}{128 \mu_{\mathrm{z}}} \frac{\Delta \mathrm{p}}{\mathrm{L}_{0}^{\mathrm{D}_{\mathrm{L}}}} \int_{\chi_{\min }}^{\chi_{\max }} \chi^{2+\mathrm{D}_{\mathrm{L}}-\mathrm{D}_{\mathrm{d}}}\left[1-\vartheta\left(1-\frac{\chi_{\mathrm{c}}}{\chi}\right)^{4}\right] \mathrm{d} \chi$

After integration, yields

$$
\begin{aligned}
& \Phi_{\mathrm{k}_{\mathrm{ow}}}=\frac{\pi \omega^{2} \mathrm{D}_{\mathrm{d}} \chi_{\max }^{\mathrm{D}_{\mathrm{d}}}}{8(3 \omega+1)\left(3 \omega+\mathrm{D}_{\mathrm{L}}-\mathrm{D}_{\mathrm{d}} \omega\right)}\left(\frac{1}{4 \mathrm{k}_{\mathrm{ow}}} \frac{\Delta \mathrm{p}}{\mathrm{L}_{0}^{\mathrm{D}_{\mathrm{L}}}}\right)^{\frac{1}{\omega}} \\
& \left(\chi_{\max }^{\frac{3 \omega+\mathrm{D}_{\mathrm{L}}-\mathrm{D}_{\mathrm{d}} \omega}{\omega}}-\chi_{\min }^{\frac{3 \omega+\mathrm{D}_{\mathrm{L}}-\mathrm{D}_{\mathrm{d}} \omega}{\omega}}\right) \\
& \Phi_{\mathrm{k}_{\mathrm{Bp}}}=\frac{\pi \mathrm{a}_{\mathrm{k}_{\mathrm{Bp}}} \mathrm{D}_{\mathrm{d}} \chi_{\max }^{\mathrm{D}_{\mathrm{d}}}}{128 \mathrm{k}_{\mathrm{Bp}}\left(3+\mathrm{D}_{\mathrm{L}}-\mathrm{D}_{\mathrm{d}}\right)\left(2+\mathrm{D}_{\mathrm{L}}-\mathrm{D}_{\mathrm{d}}\right)} \frac{\Delta \mathrm{p}}{\mathrm{L}_{0}^{\mathrm{D}_{\mathrm{L}}}} \\
& \left(\chi_{\max }^{3+\mathrm{D}_{\mathrm{L}}-\mathrm{D}_{\mathrm{d}}}-\chi_{\max }^{3+\mathrm{D}_{\mathrm{L}}-\mathrm{D}_{\mathrm{d}}}\right)
\end{aligned}
$$

with

$$
\mathrm{a}_{\mathrm{k}_{\mathrm{Bp}}}=\left[\left(2+\mathrm{D}_{\mathrm{L}}-\mathrm{D}_{\mathrm{d}}\right)-\frac{4}{3} \frac{\chi_{\mathrm{p}}}{\chi_{\max }}\left(3+\mathrm{D}_{\mathrm{L}}-\mathrm{D}_{\mathrm{d}}\right)\right]
$$

and

$$
\begin{aligned}
& \Phi_{\mu_{\mathrm{z}}}=\frac{\pi \mathrm{D}_{\mathrm{d}} \chi_{\max }^{\mathrm{D}_{\mathrm{d}}}}{128 \mu_{\mathrm{z}}} \frac{\Delta \mathrm{p}}{\mathrm{L}_{0}^{\mathrm{D}_{\mathrm{L}}}}\left[\frac{\left(\chi_{\max }^{3+\mathrm{D}_{\mathrm{L}}-\mathrm{D}_{\mathrm{d}}}-\chi_{\min }^{3+\mathrm{D}_{\mathrm{L}}-\mathrm{D}_{\mathrm{d}}}\right)}{3+\mathrm{D}_{\mathrm{L}}-\mathrm{D}_{\mathrm{d}}}+\right. \\
& \left.\mathrm{a}_{\mu_{\mathrm{z}}}\left(\frac{\vartheta \chi_{\max }^{\mathrm{D}_{\mathrm{L}}-\mathrm{D}_{\mathrm{d}}-1}}{\mathrm{a}_{0}}-\frac{\vartheta \chi_{\min }^{\mathrm{D}_{\mathrm{L}}-\mathrm{D}_{\mathrm{d}}-1}}{\mathrm{a}_{0}}\right)\right]
\end{aligned}
$$

with

$\mathrm{a}_{\mu_{\mathrm{z}}}=\mathrm{a}_{1} \chi_{\mathrm{c}}^{4}+\mathrm{a}_{2} \chi_{\mathrm{c}}^{3} \chi_{\min }+\mathrm{a}_{3} \chi_{\mathrm{c}}^{2} \chi_{\min }^{2}+\mathrm{a}_{4} \chi_{\mathrm{c}} \chi_{\min }^{3}+\mathrm{a}_{5} \chi_{\min }^{4}$

$\mathrm{a}_{0}=6\left(2+\mathrm{D}_{\mathrm{L}}-\mathrm{D}_{\mathrm{d}}\right)-5\left(2+\mathrm{D}_{\mathrm{L}}-\mathrm{D}_{\mathrm{d}}\right)^{2}-5\left(2+\mathrm{D}_{\mathrm{L}}-\mathrm{D}_{\mathrm{d}}\right)^{3}+$

$5\left(2+D_{L}-D_{d}\right)^{4}-\left(2+D_{L}-D_{d}\right)^{5}$

$\mathrm{a}_{1}=\left(2+\mathrm{D}_{\mathrm{L}}-\mathrm{D}_{\mathrm{d}}\right)^{4}-2\left(2+\mathrm{D}_{\mathrm{L}}-\mathrm{D}_{\mathrm{d}}\right)^{3}-$

$\left(2+D_{L}-D_{d}\right)^{2}+2\left(2+D_{L}-D_{d}\right)$

$\mathrm{a}_{2}=-4\left(2+\mathrm{D}_{\mathrm{L}}-\mathrm{D}_{\mathrm{d}}\right)^{4}+12\left(2+\mathrm{D}_{\mathrm{L}}-\mathrm{D}_{\mathrm{d}}\right)^{3}+$

$4\left(2+D_{L}-D_{d}\right)^{2}-12\left(2+D_{L}-D_{d}\right)$

$\mathrm{a}_{3}=6\left(2+\mathrm{D}_{\mathrm{L}}-\mathrm{D}_{\mathrm{d}}\right)^{4}-24\left(2+\mathrm{D}_{\mathrm{L}}-\mathrm{D}_{\mathrm{d}}\right)^{3}+$

$6\left(2+D_{L}-D_{d}\right)^{2}+36\left(2+D_{L}-D_{d}\right)$

$\mathrm{a}_{4}=-4\left(2+\mathrm{D}_{\mathrm{L}}-\mathrm{D}_{\mathrm{d}}\right)^{4}+20\left(2+\mathrm{D}_{\mathrm{L}}-\mathrm{D}_{\mathrm{d}}\right)^{3}-$

$20\left(2+D_{L}-D_{d}\right)^{2}-20\left(2+D_{L}-D_{d}\right)+24$

$\mathrm{a}_{5}=\left(2+\mathrm{D}_{\mathrm{L}}-\mathrm{D}_{\mathrm{d}}\right)^{4}-6\left(2+\mathrm{D}_{\mathrm{L}}-\mathrm{D}_{\mathrm{d}}\right)^{3}+$

$11\left(2+D_{L}-D_{d}\right)^{2}-6\left(2+D_{L}-D_{d}\right)$

The porosity of the bundle of capillary tubes can be expressed as

$\varepsilon=-\frac{1}{\mathrm{~L}_{0}^{3}} \int_{\chi \min }^{\chi_{\max }} \frac{\pi \chi^{2} \mathrm{~L}(\chi)}{4} \mathrm{dN}(\mathrm{L} \geq \chi)$

Integrating Eq. (24) over the entire range of pore sizes, on the basis of Eqs. (6) and (9) yields

$$
\begin{aligned}
& \varepsilon=\frac{\pi}{4 \mathrm{~L}_{0}^{3}} \mathrm{D}_{\mathrm{d}} \chi_{\text {max }}^{\mathrm{D}_{\mathrm{d}}} \mathrm{L}_{0}^{\mathrm{D}_{\mathrm{L}}} \int_{\chi \text { min }}^{\chi_{\max }} \chi^{2-\mathrm{D}_{\mathrm{d}}-\mathrm{D}_{\mathrm{L}}} \mathrm{d} \chi= \\
& \frac{\pi \mathrm{D}_{\mathrm{d}} \chi_{\max }^{\mathrm{D}_{\mathrm{d}}} \mathrm{L}_{0}^{\mathrm{D}_{\mathrm{L}}-3}}{12-4 \mathrm{D}_{\mathrm{d}}-4 \mathrm{D}_{\mathrm{L}}}\left(\chi_{\text {max }}^{3-\mathrm{D}_{\mathrm{d}}-\mathrm{D}_{\mathrm{L}}}-\chi_{\text {min }}^{3-\mathrm{D}_{\mathrm{d}}-\mathrm{D}_{\mathrm{L}}}\right)
\end{aligned}
$$

Equation (25) shows how porosity is linked with the tube fractal dimensions $D_{d}$ and $D_{L}$. Fluid flow can be expressed in terms of porosity by applying this equation into the previous equations. Substituting Eq. (25) into Eq. (21), and re-arranging yields

$$
\begin{aligned}
& \Phi_{\mathrm{k}_{\mathrm{ow}}}=\frac{\zeta_{\mathrm{k}_{\mathrm{ow}}} \omega^{2} \mathrm{D}_{\mathrm{d}} \chi_{\max }^{\frac{3 \omega+\mathrm{D}_{\mathrm{L}}}{\omega}}}{(3 \omega+1)\left(3 \omega+\mathrm{D}_{\mathrm{L}}-\mathrm{D}_{\mathrm{d}} \omega\right) \mathrm{L}_{0}^{\frac{\mathrm{D}_{\mathrm{L}}}{\omega}}} \\
& \left\{1-\left[1-\left(\frac{\mathrm{L}_{0}}{\chi_{\max }}\right)^{3-\mathrm{D}_{\mathrm{L}}} \frac{\left(12-4 \mathrm{D}_{\mathrm{d}}-4 \mathrm{D}_{\mathrm{L}}\right) \varepsilon}{\pi \mathrm{D}_{\mathrm{d}}}\right]^{\frac{3 \omega+\mathrm{D}_{\mathrm{L}}-\mathrm{D}_{\mathrm{d}} \omega}{3 \omega-\mathrm{D}_{\mathrm{d}} \omega-\mathrm{D}_{\mathrm{L}} \omega}}\right\}
\end{aligned}
$$

with

$$
\zeta_{\mathrm{k}_{\mathrm{ow}}}=\frac{\pi}{8}\left(\frac{\Delta \mathrm{p}}{4 \mathrm{k}_{\mathrm{ow}}}\right)^{\frac{1}{\omega}}
$$

Inserting the Eq. (25) into Eq. (22), we obtain

$$
\begin{aligned}
& \Phi_{\mathrm{k}_{\mathrm{Bp}}}=\frac{\zeta_{\mathrm{k}_{\mathrm{Bp}}} \mathrm{D}_{\mathrm{d}} \chi_{\max }^{3+\mathrm{D}_{\mathrm{L}}}}{\left(3+\mathrm{D}_{\mathrm{L}}-\mathrm{D}_{\mathrm{d}}\right)\left(2+\mathrm{D}_{\mathrm{L}}-\mathrm{D}_{\mathrm{d}}\right) \mathrm{L}_{0}^{\mathrm{D}_{\mathrm{L}}}} \\
& {\left[\left(2+\mathrm{D}_{\mathrm{L}}-\mathrm{D}_{\mathrm{d}}\right)-\frac{4}{3} \frac{\chi_{\mathrm{p}}}{\chi_{\max }}\left(3+\mathrm{D}_{\mathrm{L}}-\mathrm{D}_{\mathrm{d}}\right)\right]} \\
& \left\{1-\left[1-\left(\frac{\mathrm{L}_{0}}{\chi_{\max }}\right)^{3-\mathrm{D}_{\mathrm{L}}} \frac{\left(12-4 \mathrm{D}_{\mathrm{d}}-4 \mathrm{D}_{\mathrm{L}}\right) \varepsilon}{\pi \mathrm{D}_{\mathrm{d}}}\right]^{\frac{3+\mathrm{D}_{\mathrm{L}}-\mathrm{D}_{\mathrm{d}}}{3-\mathrm{D}_{\mathrm{d}}-\mathrm{D}_{\mathrm{L}}}}\right\}
\end{aligned}
$$

with

$$
\zeta_{\mathrm{k}_{\mathrm{Bp}}}=\frac{\pi \Delta \mathrm{p}}{128 \mathrm{k}_{\mathrm{Bp}}}
$$

Combining Eqs. 23 and 25, and rearranging, provides

$$
\begin{aligned}
& \Phi_{\mu_{2}}=\frac{\xi_{\mu_{2}} D_{d} \chi_{\max }^{3+D_{\mathrm{L}}}}{\mathrm{L}_{0}^{D_{\mathrm{L}}}}\left\{\frac{1}{3+\mathrm{D}_{\mathrm{L}}-\mathrm{D}_{\mathrm{d}}}\left(1-\psi_{\mu_{\mathrm{L}}}^{\frac{3+\mathrm{D}_{\mathrm{L}}-\mathrm{D}_{\mathrm{d}}}{3-\mathrm{D}_{\mathrm{L}}}}\right)+\right. \\
& \frac{\vartheta}{a_{0}}\left[a_{1}\left(\frac{\chi_{c}}{\chi_{\max }}\right)^{4}\left(1-\psi_{\mu_{z}}^{\frac{D_{L}-D_{d}-1}{3-D_{d}-D_{L}}}\right)+a_{2}\left(\frac{\chi_{c}}{\chi_{\max }}\right)^{3}\left(1-\psi_{\mu_{z}}^{\frac{D_{L}-D_{d}}{3-D_{d}-D_{L}}}\right)+\right. \\
& \mathrm{a}_{3}\left(\frac{\chi_{\mathrm{c}}}{\chi_{\text {max }}}\right)^{2}\left(1-\psi_{\mu_{\mathrm{L}}}^{\frac{1+\mathrm{D}_{\mathrm{L}}-\mathrm{D}_{\mathrm{d}}}{3-\mathrm{D}_{\mathrm{L}}-\mathrm{D}_{\mathrm{L}}}}\right)+\mathrm{a}_{4}\left(\frac{\chi_{\mathrm{c}}}{\chi_{\max }}\right)\left(1-\psi_{\mu_{\mathrm{L}}}^{\frac{2+\mathrm{D}_{\mathrm{L}}-\mathrm{D}_{\mathrm{d}}}{3-\mathrm{D}_{\mathrm{L}}}}\right)+ \\
& \left.\left.a_{5}\left(1-\psi_{\mu_{\mu_{2}}}^{\frac{3+D_{L_{2}}-D_{d}}{3-D_{L_{L}}}}\right)\right]\right\}
\end{aligned}
$$

with

$\xi_{\mu_{\mathrm{z}}}=\frac{\pi \xi_{\mu_{\mathrm{z}}} \Delta \mathrm{p}}{128 \mu_{\mathrm{z}}}$

$\psi_{\mu_{z}}=1-\left(\frac{L_{0}}{\chi_{\max }}\right)^{3-D_{L}} a_{6} \varepsilon$ 
and

$\mathrm{a}_{6}=\frac{\left(12-4 \mathrm{D}_{\mathrm{d}}-4 \mathrm{D}_{\mathrm{L}}\right) \varepsilon}{\pi \mathrm{D}_{\mathrm{d}}}$

Equations (26) to (28) describe the flow in terms of fluid properties $(\mu, \mathrm{k}, \omega)$, size of tubes $\left(\chi_{\max }, \mathrm{L}_{0}\right)$, and fractal dimensions $\left(D_{L}, D_{d}\right)$.

\subsection{Permeability of a Fractal Porous Material}

The Darcy equation is the standard approach to characterize flows through porous materials, in which the inertial forces can be neglected with respect to the viscous forces. In this equation a global index (i.e., the permeability that represents the ability of the material to transmit the fluid through it), relates the fluid flow with the pressure drop through the material (Larson 1981)

$$
\left(\frac{\Phi}{\mathrm{A}}\right)^{\omega}=\frac{\mathrm{K}}{\mathrm{k}_{\mathrm{ow} / \mathrm{Bp} / \mu}} \frac{\Delta \mathrm{p}}{\mathrm{L}}
$$

Here $\mathrm{K}$ is the permeability of the porous material, and its usage is restricted to the occurrence of viscous forces, that are the relevant forces for flows described by Eqs. (26) to (28). So, comparing Eq. (29) to Eqs. (26) to (28) yields

$$
\begin{aligned}
& \mathrm{K}_{\mathrm{k}_{\mathrm{ov}}}^{*}=\left[\frac{\pi \omega^{2} \mathrm{D}_{\mathrm{d}}}{2^{\frac{3 \omega+2}{\omega}}(3 \omega+1)\left(3 \omega+\mathrm{D}_{\mathrm{L}}-\mathrm{D}_{\mathrm{d}} \omega\right)}\right]^{\omega} \\
& \left\{1-\left[1-\left(\frac{\mathrm{L}_{0}}{\chi_{\max }}\right)^{3-\mathrm{D}_{\mathrm{L}}} \frac{\left(12-4 \mathrm{D}_{\mathrm{d}}-4 \mathrm{D}_{\mathrm{L}}\right) \varepsilon}{\pi \mathrm{D}_{\mathrm{d}}}\right]^{\frac{3 \omega+\mathrm{D}_{\mathrm{L}}-\mathrm{D}_{\mathrm{d}} \omega}{3 \omega-\mathrm{D}_{\mathrm{d}} \omega-\mathrm{D}_{\mathrm{L}} \omega}}\right\}^{\omega} \\
& \mathrm{K}_{\mathrm{k}_{\mathrm{BP}}}^{*}=\frac{\pi \mathrm{D}_{\mathrm{d}}}{128\left(3+\mathrm{D}_{\mathrm{L}}-\mathrm{D}_{\mathrm{d}}\right)\left(2+\mathrm{D}_{\mathrm{L}}-\mathrm{D}_{\mathrm{d}}\right)} \\
& {\left[\left(2+D_{L}-D_{d}\right)-\frac{4}{3} \frac{\chi_{p}}{\chi_{\max }}\left(3+D_{L}-D_{d}\right)\right]} \\
& \left\{1-\left[1-\left(\frac{\mathrm{L}_{0}}{\chi_{\max }}\right)^{3-\mathrm{D}_{\mathrm{L}}} \frac{\left(12-4 \mathrm{D}_{\mathrm{d}}-4 \mathrm{D}_{\mathrm{L}}\right) \varepsilon}{\pi \mathrm{D}_{\mathrm{d}}}\right]^{3+\mathrm{D}_{\mathrm{L}}-\mathrm{D}_{\mathrm{d}}}\right\} \\
& \mathrm{K}_{\mathrm{k}_{\mu z}}^{*}=\frac{\pi \mathrm{D}_{\mathrm{d}}}{128}\left\{\frac{1}{3+\mathrm{D}_{\mathrm{L}}-\mathrm{D}_{\mathrm{d}}}\left(1-\psi_{\mu_{\mathrm{z}}}^{\frac{3+\mathrm{D}_{\mathrm{L}}-\mathrm{D}_{\mathrm{d}}}{3-\mathrm{D}_{\mathrm{L}}}}\right)+\right. \\
& \frac{\vartheta}{a_{0}}\left[a_{1}\left(\frac{\chi_{c}}{\chi_{\text {max }}}\right)^{4}\left(1-\psi_{\mu_{L}}^{\frac{D_{L}-D_{d}-1}{3-D_{d}-D_{L}}}\right)+a_{2}\left(\frac{\chi_{c}}{\chi_{\text {max }}}\right)^{3}\left(1-\psi_{\mu_{L}}^{\frac{D_{L}-D_{d}}{3-D_{d}-D_{L}}}\right)+\right. \\
& \mathrm{a}_{3}\left(\frac{\chi_{\mathrm{c}}}{\chi_{\text {max }}}\right)^{2}\left(1-\psi_{\mu_{\mathrm{L}}}^{\frac{1+\mathrm{D}_{\mathrm{L}}-\mathrm{D}_{\mathrm{d}}}{3-\mathrm{D}_{\mathrm{L}}}}\right)+\mathrm{a}_{4}\left(\frac{\chi_{\mathrm{c}}}{\chi_{\text {max }}}\right)\left(1-\psi_{\mu_{\mathrm{L}}}^{\frac{2+\mathrm{D}_{\mathrm{L}}-\mathrm{D}_{\mathrm{d}}}{3-\mathrm{D}_{\mathrm{L}}}}\right)+ \\
& \left.\left.a_{5}\left(1-\psi_{\mu_{\mu_{2}}-D_{d}}^{\frac{3+D_{L}-D_{d}}{3-D_{L}}}\right)\right]\right\}
\end{aligned}
$$

with

$$
\mathrm{K}_{\mathrm{k}_{\mathrm{ow}}}^{*}=\frac{\mathrm{K}_{\mathrm{k}_{\mathrm{ow}}}}{\left(\frac{\chi_{\max }^{3 \omega+\mathrm{D}_{\mathrm{L}}}}{\mathrm{L}_{0}^{2 \omega+\mathrm{D}_{\mathrm{L}}-1}}\right)}
$$

$$
\mathrm{K}_{\mathrm{k}_{\mathrm{Bp}}}^{*}=\frac{\mathrm{K}_{\mathrm{k}_{\mathrm{Bp}}}}{\left(\frac{\chi_{\max }^{3+\mathrm{D}_{\mathrm{L}}}}{\mathrm{L}_{0}^{+\mathrm{D}_{\mathrm{L}}}}\right)}
$$

$$
\mathrm{K}_{\mathrm{k}_{\mu z}}^{*}=\frac{\mathrm{K}_{\mathrm{k}_{\mathrm{\mu z}}}}{\left(\frac{\chi_{\max }^{3+\mathrm{D}_{\mathrm{L}}}}{\mathrm{L}_{0}^{++\mathrm{D}_{\mathrm{L}}}}\right)}
$$

where $\mathrm{K}_{\mathrm{k}_{\mathrm{ow}}}^{*}, \mathrm{~K}_{\mathrm{k}_{\mathrm{pp}}}^{*}$ and $\mathrm{K}_{\mathrm{k}_{\mathrm{\mu z}}}^{*}$ are the dimensionless permeabilities for power-law fluids, Bingham plastic fluids, and marginal zone models, respectively. These equations reveal that the dimensionless permeability depends not only on the porosity and fractal dimensions $\mathrm{D}_{\mathrm{d}}$ and $\mathrm{D}_{\mathrm{L}}$, but also on the specific characteristics of the fluid model that is adopted.

\section{RESUltS AND DISCUSSION}

In order to understand the interplay between porous material and fluid flow, the effect of porosity, fractal dimensions, and other geometry parameters are examined.

The resistance to fluid flow can be defined as $\mathrm{R}=\left(\frac{\Delta \mathrm{p}}{\mathrm{L}}\right)^{\frac{1}{\omega}} \int_{\phi(\chi)}$. Resistances of tortuous capillary tubes defined according to Eqs. (14) to (16) are depicted at Figs. 2a to 2c. For power-law fluid (Fig. 2a), the flow resistance increases with the $\mathrm{k}_{\mathrm{o}, \mathrm{w}}$ and $\mathrm{D}_{\mathrm{L}}$ but decreases with $\omega$ and $\chi$. In thermodynamic terms, higher consistency index and fractal dimension means higher energy dissipation, and higher fluid behavior index and tube diameter less energy dissipation. This result is in line with general theory for fluid flow through tubes (Miguel 2019).

According to Fig. 2b, resistance to Bingham plastic fluid flow increases with $\mathrm{k}_{\mathrm{Bp}}, \chi_{\mathrm{p}} / \chi$ and $\mathrm{D}_{\mathrm{L}}$ but decreases with $\chi$. This result agrees with the trend observed for the power law fluid. Notice that the flow resistance is only slightly dependent on ratio between the plug diameter and the tube diameter $\left(\chi_{\mathrm{p}} / \chi\right)$. This can be explained due to the fact that diameter of the plug is much lower than the diameter of the tube.

Flow resistance with Fåhræus-Lindqvist effect (Fig. 2c) increases with the $\mu_{\mathrm{z}}, v$ and $D_{\mathrm{L}}$ but decreases both with $\chi_{\mathrm{c}} / \chi$ and the size of tube $\chi$. This can be explained due to the fact that diameter of the marginal zone is much lower than the diameter of the tube. Note that a higher $\chi_{\mathrm{c}}$ means concentration of red blood cells scattered over the tube diameter, and less energy dissipation.

According to Eq. 14, both Newtonian and power law fluid flows have the same flow resistance when the ratio between tube dimensions is

$\frac{\chi_{N}}{\chi_{\omega}}=\left[\frac{16}{(3 \omega+1)}\left(\frac{\mu_{N}^{\omega}}{4 \mathrm{k}_{\mathrm{ow}}}\right)^{\frac{1}{\omega}} \chi_{\omega}^{\frac{\mathrm{D}_{\mathrm{L}}-\omega \mathrm{D}_{\mathrm{L}}}{\omega}}\right]^{\frac{1}{3+\mathrm{D}_{\mathrm{L}}}}$ 


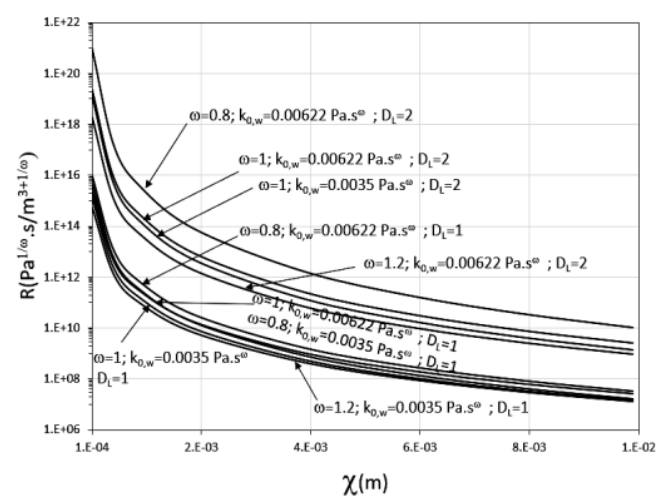

Fig. 2a. Flow resistance of a tortuous capillary tube for power-law fluids.

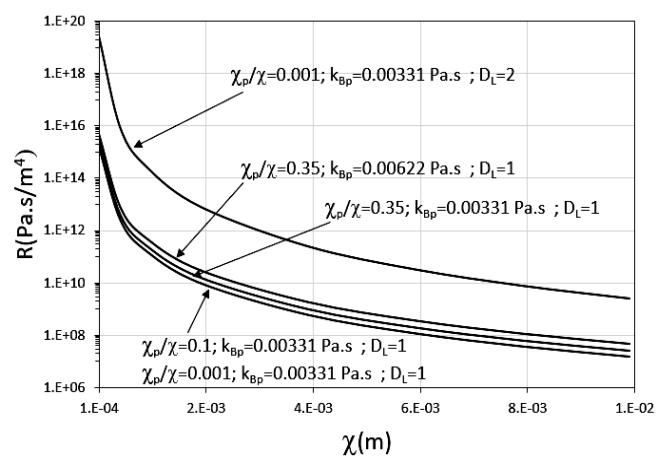

Fig. 2b. Flow resistance of a tortuous capillary tube for Bingham plastic fluids.

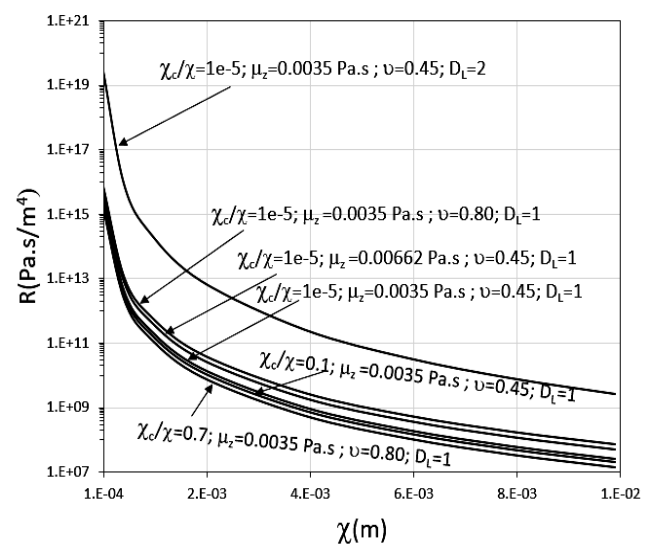

Fig. 2c. Flow resistance of a tortuous capillary tube considering the marginal zone model.

The same resistance occurs in tubes with flows of Newtonian and Bingham plastic fluids (Eqs. 14 and 15) and flows of Newtonian and marginal zone models (Eqs. 14 and 16) when the ratio between tube dimensions are

$$
\begin{aligned}
& \frac{\chi_{\mathrm{N}}}{\chi_{\mathrm{Bp}}}=\left[\frac{\mu_{\mathrm{N}}}{\mathrm{k}_{\mathrm{Bp}}}\left(1-\frac{4}{3} \frac{\chi_{\mathrm{p}}}{\chi_{\mathrm{Bp}}}\right)\right]^{\frac{1}{3+\mathrm{D}_{\mathrm{L}}}} \\
& \frac{\chi_{\mathrm{N}}}{\chi_{\mathrm{z}}}=\left\{\frac{\mu_{\mathrm{N}}}{\mu_{\mathrm{z}}}\left[1-\vartheta\left(1-\frac{\chi_{\mathrm{c}}}{\chi_{\mathrm{z}}}\right)^{4}\right]\right\}^{\frac{1}{3+\mathrm{D}_{\mathrm{L}}}}
\end{aligned}
$$

Here the subscripts $\mathrm{N}, \omega, \mathrm{Bp}$ and $\mathrm{z}$ mean Newtonian, power-law, Bingham plastic and marginal zone models, respectively. These results allow us to compare the size of the tube diameters to have the same conditions for energy dissipation.

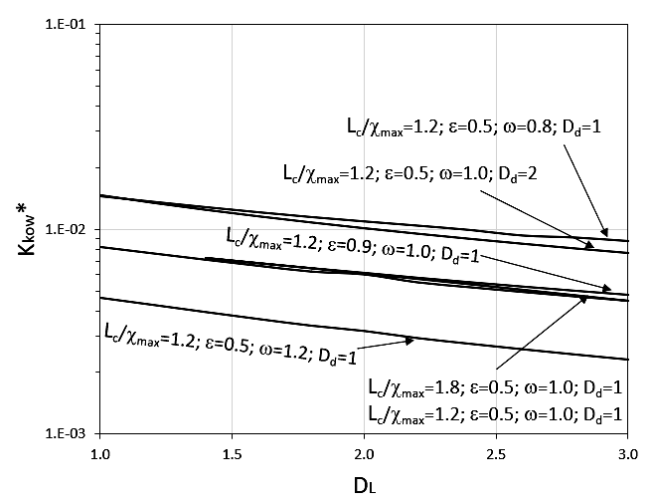

Fig. 3a. Dimensionless permeability of prefractal tube bundle for flows of power-law fluids.

Figures $3 \mathrm{a}$ to $3 \mathrm{c}$ plot the dimensionless permeability based on models presented at section 3.3. For flows of power-law fluids (Fig. 3a) the dimensionless permeability increases with $D_{d}$ but decreases with $\mathrm{D}_{\mathrm{L}}$ and $\omega$. Notice $\mathrm{L}_{\mathrm{o}} / \chi_{\max }$ has a minor effect on $\mathrm{K}_{\mathrm{kow}}^{*}$.

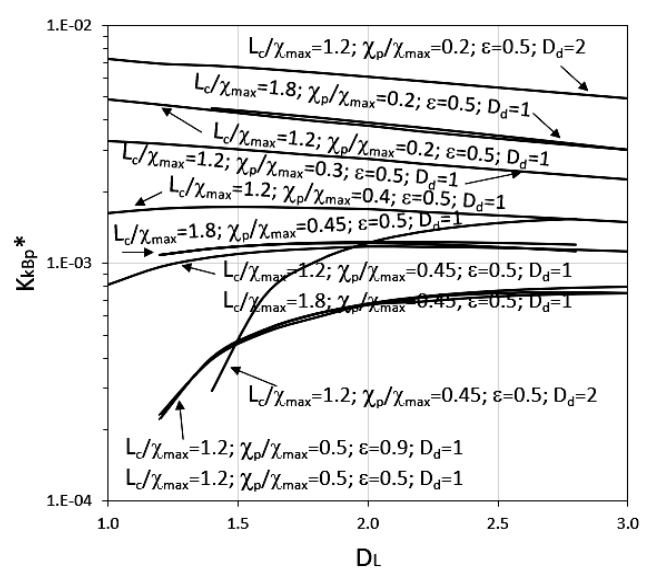

Fig. 3b. Dimensionless permeability of prefractal tube bundle for flows of Bingham plastic fluids.

For flows of Bingham plastic fluids (Fig. 3b) we can see that

- $\chi_{\mathrm{p}} / \chi_{\max } \geq 0.4: \mathrm{K}_{\mathrm{kBp}}^{*}$ increases with $\varepsilon, \mathrm{D}_{\mathrm{d}}$ and $\mathrm{Lo} / \chi_{\max }$. For $\chi_{\mathrm{p}} / \chi_{\max } \geq 0.47, \mathrm{~K}_{\mathrm{kBp}}^{*}$ increases with $\mathrm{D}_{\mathrm{L}}$ but for $0.4<\chi_{\mathrm{p}} / \chi_{\max }<0.47, \mathrm{~K}_{\mathrm{kBp}}^{*}$ increases with $D_{L}$ until $D_{L}=2$, and subsequently decreases with $\mathrm{D}_{\mathrm{L}}$,
$\chi_{\mathrm{p}} / \chi_{\max }<0.4: \mathrm{K}_{\mathrm{kBp}}^{*}$ increases with $\varepsilon, \mathrm{D}_{\mathrm{d}}$ and

$\mathrm{L}_{\mathrm{o}} / \chi_{\max }$ but decreases with $\mathrm{D}_{\mathrm{L}}$. 


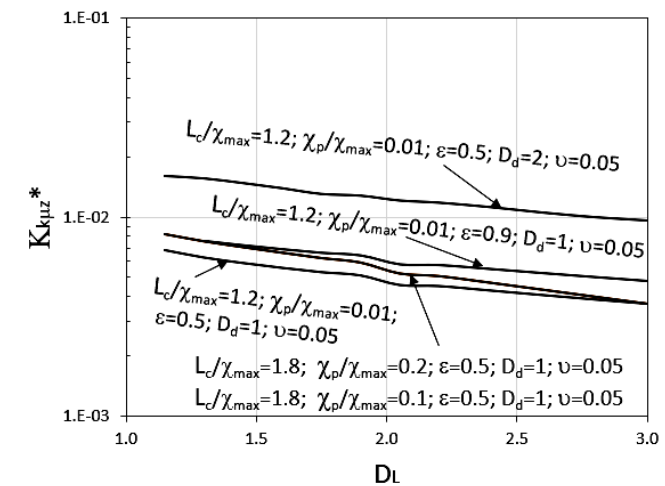

Fig. 3c. Dimensionless permeability of prefractal tube bundle considering the marginal zone of model.

Figure $3 \mathrm{c}$ shows that the $\mathrm{K}_{\mathrm{k \mu z}}^{*}$ increases with $\varepsilon$, $\mathrm{D}_{\mathrm{d}}$ and $\mathrm{Lo}_{\mathrm{o}} / \chi_{\max }$ but decreases with $\mathrm{D}_{\mathrm{L}}$. There is a minor influence of $\chi_{\mathrm{c}} / \chi_{\max }$ and $v$ on the dimensionless permeability $\mathrm{K}_{\mathrm{k \mu z}}^{*}$.

\section{CONCLUSION}

Evaluation of permeability of a prefractal bundle of capillary tubes is significant for many applications, and thus of importance in multi-disciplinary fields. Here, fractal dimensions for pore size distribution and capillary tortuosity are accounted to characterize the pore-scale structure. The porosity in terms of fractal dimensions for pore size distribution is obtained.

For blood flow in larger vessels, a Newtonian model constitute a good approximation for blood, but in smaller vessels, the non-Newtonian behavior manifests and must be accounted. Models for flows of power law fluid, Bingham plastic fluid and marginal zone theory model are considered. Generalized models for porous media composed by prefractal tube bundles are rigorously obtained. In addition, models for flow resistances through single tortuous tubes, and the relationship between fluid velocity through tortuous tubes and through straight tubes, in terms of fractal dimensions are also presented. All the parameters in these models have a clear physical meaning, and capture microstructure characteristics and essential physical features that occur within the capillary tubes.

\section{ACKNOWLEDGEMENTS}

This work was supported by the project ALT20-030145-FEDER-029624 and by the Portuguese Foundation for Science and Technology (FCT) project UIDB/04683/2020 - ICT (Institute of Earth Sciences

\section{REFERENCES}

Adler, P. M. (1996). Transports in fractal porous media. Journal of Hydrology 187, 195-213.
Bejan, A., I. Dincer, S. Lorente, A. F. Miguel and A. Reis (2004) Porous and Complex Flow Structures in Modern Technologies. Springer, New York, USA.

Bird, R. B., W. E. Stewart and E. N. Lightfoot (1960). Transport Phenomena. John Wiley \& Sons, New York, USA.

Bittner, H. R. (1991). Modelling of fractal vessel systems. In H. O. Peitgen, J. M. Henriques, and L. F. Penedo (Ed.). Fractals in the Fundamental and Applied Sciences, Elsevier, Amsterdam, Netherlands, 47-68.

Cai, J., L. Luo, R. Ye, X. Zeng and X. Hu (2015). Recent advances on fractal modeling of permeability for fibrous porous media. Fractals 23, 1540006.

Chen, X., T. Li, J. Shen and Z. Hu (2016) Fractal design of microfluidics and nanofluidics-a review. Chemometrics and Intelligent Laboratory Systems 155, 19-25.

Delalat, B., C. Cozzi, S. R. Ghaemi, G. Polito, F. H. Kriel, T. D. Michel, F. J. Harding, C. Priest, G. Barillaro and N. H. Voelcker (2018). Microengineered bioartificial liver chip for drug toxicity screening. Advanced Functional Materials 28, 1801825.

Dougherty, G. and J. Varro (2000). A quantitative index for the measurement of the tortuosity of blood vessels. Medical Engineering \& Physics 22, 567-574.

Fåhræus, R. and T. Lindqvist (1931). The viscosity of the blood in narrow capillary tubes. Am. $J$. Physiol. 96, 562-568.8

Fortier, A., V. Gullapalli and R. A. Mirshams (2014). Review of biomechanical studies of arteries and their effect on stent performance. IJC Heart \& Vessels 4, 12-18.

Fung, Y. C. (1993) Biomechanics: Circulation. Springer-Verlag, New York, USA.

Hansen, J. P. and A. T. Skjeltorp (1988). Fractal pore space and rock permeability implications. Physical Review B 38, 2635-2638.

Haynes, R. H. (1960) Physical basis of the dependence of blood viscosity on tube radius. The American Journal of Physiology 198, $1193-1200$

Illa, X., S. Vila, J. Yeste, C. Peralta, J. GraciaSancho and R. Villa (2014). A Novel modular bioreactor to in vitro study the hepatic sinusoid. PLoS One 9, e111864.

Jacquin, C. G. and P. M. Adler, (1987). Fractal porous media II: geometry of porous geological structures. Transp. Porous Media 2, 571-596.

Katz, A. J. and A. H. Thompson (1985). Fractal sandstone pores: implications for conductivity and pore formation. Physical Review Letters 54, 1325-1328. 
A. F. Miguel / JAFM, Vol. 13, No. 6, pp. 1675-1682, 2020.

Krohn, C. E. (1988). Fractal measurements of sandstones, shales, and carbonates. Journal of Geophysical Research: Solid Earth 93, 32973305.

Larson, R. G. (1981) Derivation of generalized Darcy equations for creeping flow in porous media. Industrial \& Engineering Chemistry Fundamentals 20, 132-137.

Lei, G., N. Cao, B. J. McPherson, Q. Liao and W. Chen (2019). A novel analytical model for pore volume compressibility of fractal porous media. Scientific Reports 9, 14472.

Masters, B. R. (2004). Fractal analysis of the vascular tree in the human retina. Annual Review of Biomedical Engineering 6, 427-452.

Miguel, A. F. (2010). Fluid flow in tree-shaped constructal networks: porosity, permeability and inertial parameter. Defect and Diffusion Forum 297-301, 408-412.

Miguel, A. F. (2016). Toward an optimal design principle in symmetric and asymmetric tree flow networks. Journal of Theoretical Biology 389, 101-109.

Miguel, A. F. (2019) Towards methodologies for optimal fluid networks design. Journal of Applied Fluid Mechanics 12, 1223-1229

Miguel, A. F., R. Rosa and A. M. Silva (2000). Fractal geometry description of the permeability of a natural fissured rock. In V. Fassina (Ed.), Proceedings of 9th International Congress on Deterioration and Conservation of Stone, Elsevier Science, volume 1, 595-560.

Park, J., F. Berthiaume, M. Toner, M. L. Yarmush and A. W. Tilles (2005). Microfabricated grooved substrates as platforms for bioartificial liver reactors. Biotechnol. Bioeng. 90, 632-
644.

Pitchumani, R. and B. Ramakrishnan (1999). A fractal geometry model for evaluating permeabilities of porous preforms used in liquid composite molding. International Journal of Heat Mass Transfer 42, 2219-2232.

Pries, A. R, D. Neuhaus and P. Gaehtgens (1992). Blood viscosity in tube flow: dependence on diameter and hematocrit. The American Journal of Physiology: Heart and Circulatory Physiology 263, H1770-H1778.

Sandau, K. and H. Kurz (1997). Measuring fractal dimension and complexity - an alternative approach with an application. Journal of Microscopy 186, 164-176.

Tan, X. H., M. Q. Kui, X. P. Li, Z. L. Mao and H. Xiao (2017). Permeability and porosity models of bi-fractal porous media. International Journal of Modern Physics B 31, 1750219.

Tana, X. H., X. P. Li, J, Y. Liu, G. D. Zhang and L. H. Zhang (2014). Analysis of permeability for transient two-phase flow in fractal porous media. Journal of Applied Physics 115, 113502.

Wei, W., J. Cai, X. Hu and Q. Han (2015). An electrical conductivity model for fractal porous media. Geophysical Research Letters 42, 4833-4840.

Yu, B. M. and P. Cheng (2002). A Fractal model for permeability of bi-dispersed porous media. International Journal of Heat Mass Transfer 45, 2983-2993.

Yun, M., B. Yu, J. Lu and W. Zheng (2010). Fractal analysis of Herschel-Bulkley fluid flow in porous media. International Journal of Heat Mass Transfer 53, 3570-3574. 